\title{
Supporting Opportunistic Search in Meetings with Tangible Tabletop
}

\author{
Nan Li \\ EPFL \\ 1015 Lausanne \\ Switzerland \\ nan.li@epfl.ch
}

$\begin{array}{ll}\text { Omar Mubin } & \text { Pierre Dillenbourg } \\ \text { EPFL } & \text { EPFL } \\ 1015 \text { Lausanne } & 1015 \text { Lausanne } \\ \text { Switzerland } & \text { Switzerland } \\ \text { omar.mubin@epfl.ch } & \text { pierre.dillenbourg@epfl.ch }\end{array}$

Copyright is held by the author/owner(s).

CHI'12, May 5-10, 2012, Austin, TX, USA

ACM 978-1-4503-1016-1/12/05.
Frederic Kaplan

EPFL

1015 Lausanne

Switzerland

frederic.kaplan@epfl.ch

pierre.dillenbourg@epfl.ch

\begin{abstract}
Web searches are often needed in collocated meetings.

Many research projects have been conducted for

supporting collaborative search in information-seeking meetings, where searches are executed both intentionally and intensively. However, for most common meetings,

Web searches may happen randomly with low-intensity. They neither serve as main tasks nor major activities. This kind of search can be referred to as opportunistic search. The area of opportunistic search in meetings has not yet been studied. Our research is based upon this motivation. We propose an augmented tangible tabletop system with a semi-ambient conversation-context-aware surface as well as foldable paper browsers for supporting opportunistic search in collocated meetings. In this paper, we present our design of the system and initial findings.
\end{abstract}

\section{Author Keywords}

Paper interface; foldable display; semi-ambient display; tangible tabletop; opportunistic Web search

\section{ACM Classification Keywords}

H.5.3 [Information Interfaces and Presentation (e.g.,

$\mathrm{HCl}$ )]: Group and Organization Interfaces -

Computer-supported cooperative work. 


\section{General Terms}

Design, Human Factors

\section{Introduction}

Meetings are mostly task-oriented, and outcomes are desired. Common meeting tasks are information sharing, idea capturing, problem solving and decision making. Depending on concrete tasks, web search is not necessarily the main activity of a meeting. Web search may be required in situations when people are uncertain about terms that are mentioned in the conversation or written in paper documents. These searches are neither prepared nor predefined before a meeting. They happen opportunistically, and may last just for a short while. After one or several searches, the group returns to the main discussion. Search results are not treated as final outcome, but as mediator that facilitates meeting conversation by providing the group with certain up-to-date relevant information about their discussion. This kind of search is rather a temporary sub-activity alongside the meeting process, and we call it opportunistic search. It can be formally defined as temporary search activities conducted on opportunistically discovered content during a meeting. In contrast, traditional collaborative search often refers to intensive co-search activities with joint-effort playing a major role in e.g. information-seeking meeting scenarios.

\section{Related Work}

A number of research projects have investigated collaborative Web search interfaces. CoSearch[1] allowed group search to be conducted with a single PC and several mobile devices in parallel. TeamSearch [4] focused on querying tagged image database by combining boolean search tokens. WeSearch[3] and WebSurface[6] both

aimed at providing a tabletop environment for conducting efficient collaborative Web search tasks. All these systems focused on intensive Web search during meetings. Many features were designed to address collaborative issues such as awareness, division of labor, sharing and collaboration etc. We think such systems can not be adapted to facilitate meetings with opportunistic search for two reasons. First, opportunistic search is often light-weight, context-related and requires loose collaboration, so the aforementioned collaborative issues are not of great concern. Most functionalities that are offered to address these issues become redundant, resulting in increased cognitive load of users. Second, searches can only be executed explicitly with input devices, which may be intrusive to the on-going meeting process. As a result, how to design opportunistic search systems needs to be explored. Our contributions build upon these attempts. In this paper, we present two system design principles together with our current working prototype.

\section{Design Principles}

\section{Provide automatic search suggestions in the} background

Since the aim of opportunistic search is to facilitate the meeting process, the system should ensure a search to be fast executed in a non-intrusive way. It is preferred if search suggestions could be made based on the on-going discussion. We find conversational speech of great potential for recommending Web searches, because it is highly related to the meeting topic. We suggest a supporting system should automatically capture the conversational contexts and visualize them on a display for on-demand search. The display should be neither intrusive to the working flow of a meeting nor completely invisible in the environment. It must be semi-ambient, which has the properties of both being in the background of the collaboration process while at the same time remaining visible for the users [2]. 


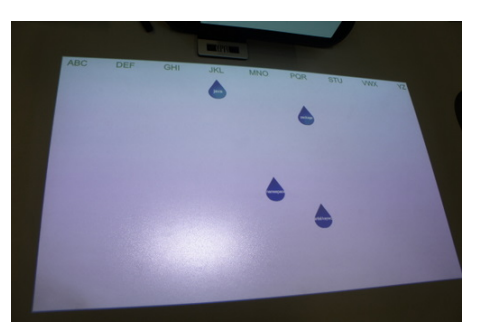

Figure 1: The semi-ambient display.
Provide Plug-and-Play Web browsing experience Browsers are required when searches are executed, but they are not needed while the group are focusing on the main task. We envision the system have browsers which work in a on-demand basis, i.e. Web browsers should have the possibility to be seamlessly plugged in/out of the discussion. Also, bookmark and history may also be useful because the former provides a auto-logged overview of activities for the group while the latter offers users the capability to save a link and revisit it later.

\section{System Design}

Our design adopts a top-projection-based augmented tabletop surface for displaying semi-ambient search suggestions. This decision was made for two reasons. First, a top-projection system can be easily deployed in most existing working environment. Second, informed by prior works (e.g. PaperLens[5] and TinkerSheets[8]), a top-projector can not only provide a large interactive surface, but also create projections for flexible displays, which in our case could be browsers. Therefore, a single projector can produce interactive space for both semi-ambient surface and paper browser displays. Nevertheless, the browsers and surface are loosely-coupled. Due to its tangibility, a paper browser can be easily "plugged" in or out of the surface, satisfying the second design principle.

Our system is built on the TinkerLamp, which is an augmented tabletop environment[7]. It is composed of a camera, a projector $(1280 \times 768$ pixels $)$ and a mirror. The mirror is facing the table at approximately one meter height to increase the projector-to-screen distance . Projection surface is of 73 by 45 centimeters. The lamp is able to track fiducial markers thanks to a fiducial-tracking library that was developed at our lab. In addition to the original TinkerLamp setup, we fixed an infrared camera on top. This camera is used to track touch-sensitive infrared pens, which work as input device for the paper browsers.

\section{Interaction Design}

The Semi-ambient Display

As aforementioned, the semi-ambient display should always display conversational context in the background, providing for opportunistic search. Before designing the system, a few issues have to be considered. First, what does "conversational context" mean exactly and what should our system employ? The context could be conversational keywords, semantic word associations, randomly grouped phrases, Web links of automatically searched results, Website snapshots or relevant pictures or documents. All these types of context, if designed properly, have the potential to foster opportunistic search. Second, how can we visualize real-time information on the tabletop surface? Should the information continuously pop up randomly, move along a fixed pattern or be grouped in a certain organized way? Our initial design only uses recognized spoken keywords. For the visualization, we have tried different styles, and currently we adopt the rain drop metaphor: real-time conversational keywords are enclosed in rain drops and fall down from the upper border (Fig. 1) at constant speed. These rain drops are arranged according to alphabetic order of the words contained. A rain drop is highlighted when the word it contains is repeated for catching users' special attention. Before a rain drop reaches the bottom side of the display, it is selectable. Afterwards, the drop disappears from the scene. Multiple words can be combined together for a single search. Details about picking words for search will be given in the next subsections. 


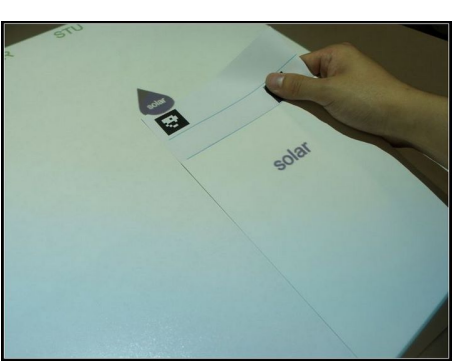

(a) Words selection

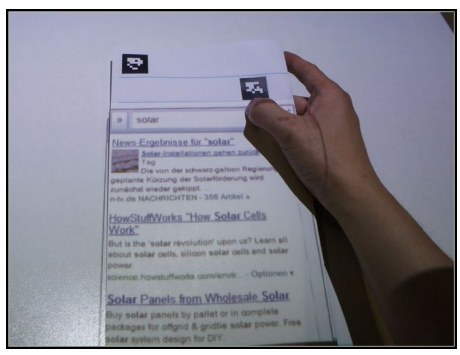

(b) Search execution

Figure 2: Selecting words for search

\section{Paper Browsers}

The system is designed to allow users make individual search queries concurrently. Due to space constraints, the system can only host at most 3 users. We designed paper browsers with borders in 3 different colors for ownership identification. A paper browser is an A4 size paper extended with an additional marker zone. It has two sides, each with four fiducial markers printed.Interactive content can be projected onto the paper sheet. For each paper sheet, the browser is on recto side, and the verso side contains a history log and Web bookmarks region. The history log consists of automatically logged Web URLs whenever a new page is loaded and URLs are categorized by word tokens that were used for initializing the query.

Users don't have to always remember which side is which. A paper browser can be used with either side facing upwards for browsing, and the other side implicitly contains the history log.

The positions of markers are designed with human ergonomics in mind. The upper part in a paper sheet is less likely to be occluded by human body during the interaction, so the marker zone is placed on top. The shape and size of a paper browser are pre-coded in the fiducial markers, so that the system knows where it is and its current form. The two topmost markers are printed on corners, and the region in between is left blank for displaying interaction feedback, such as commands issued by gestures or input devices. The other two markers are placed near the center of the lower part in the marker zone. The rest region is left blank for performing pen gestures, because these are areas where users can interact without occluding the markers. There are three categories of interaction techniques for paper browsers, we will give details respectively.
Paper browser interacting with semi-ambient display In order to select words for search, a user should use a folded paper browser to intercept a dropping word with his/her thumb pressing on the lower marker and holding the paper below the drop. Multiple words can be intercepted by the same gesture. Users can use either side of the half page with either hand, depending on his/her preference. Once a word rain drop is intercepted, it stops in the scene and changes to the user's color(Fig. 2(a)).

This is a way of providing awareness of word selection to the users. When a user removes his/her thumb from the marker, the query is finalized and the search result (currently we use Google for search) is displayed in the folded browser (Fig. 2(b)).

Peripheral input devices interacting with paper browser Although the semi-ambient surface proactively providing conversation-generated search tokens, people still may have the need to make instant explicit search queries. Since search activities in general are not intense in our scenario, the current system provides one keyboard for entering search directly. The keyboard is augmented with two fiducial markers. It can be "connected" to any paper browser by placing the keyboard on top of it. The browser is then highlighted to show connection feedback to the user (Fig. 3(a)). Once the keyboard is connected, typed words are displayed on the top of the corresponding paper browser as feedback, and the search query is executed when a user hits the "Enter" key.

The infrared pens usually work as pointing devices that are used to scroll the Web browser and click on Web links. In addition, they can also be used to perform gesture in the gesture region of a paper browser. By flipping the pen tip from left to right or vice versa, the browser navigates forwards or backwards, and corresponding text feedback is 


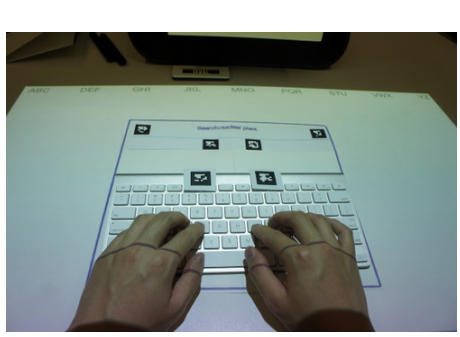

(a) Keyboard connection

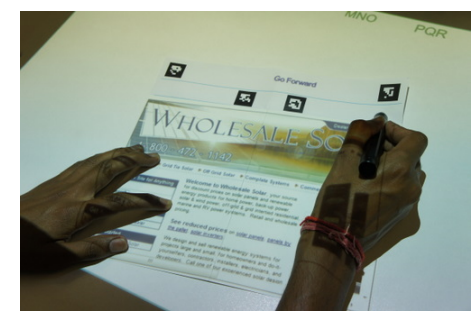

(b) Pen gestures

Figure 3: Interacting with input device

\section{shown in on the top (Fig. 3(b))}

Folding interaction with paper browser

Paper browsers, due to their material nature, are foldable. Folding and normal state can be differentiated by the system by examining the active markers in the scene. We have already described one case when folding is useful, i.e. intercepting words on the semi-ambient display. Folded paper has increased rigidity, so that it is easier to grab and hold. Another benefit of folding the display is to change its size (See Fig. 4(a)). This is especially useful in our system, since the projection surface is not large enough to accommodate more than two paper displays without occlusion. Resizing the paper browser to a smaller size whenever needed, is a solution to avoid occlusion. However, it is difficult to legibly display small text, due to resolution constraints of the projector. So half-sized browsers still use the same resolution of the Webpage. Since half-sized browsers are mostly used when executing search queries by interacting with the semi-ambient display, we use the PDA version of Google website, which perfectly fits the half-sized display without the need of horizontal scrolling. When a user clicks a link, the display can be unfolded, calling for shared attention of the other participants.

Folding can also be interpreted as meaningful gestures. A history URL is implicitly saved and kept private, but a bookmark is explicitly saved and shared. Thinking of reading a book, a page can be marked by turning down its corner. The bookmark function of our paper browser adopts this metaphor. The top-right corner can be turned down to add the current Webpage into the bookmark(See Fig. 4(b)).

\section{Informal User Study}

To find out how our system may help groups with opportunistic search during meetings, we conducted several informal user studies. 12 participants (4 groups, each containing 3 users aged between 25 and 45) were recruited to test the system in a non-search-intensive decision-making scenario. Instead of a real speech recognizer, we used WizardOfOZ method by employing a person to enter keywords spoken in the conversation into the system. The rule is that only original forms of nouns, verbs, adjectives and adverbs are entered. A list of 300 preselected stop words were omitted by the system automatically.

We asked each participant to give feedback in a post-test questionnaire regarding the following aspects: (1) unobtrusiveness of the system, which corresponds to how they conduct Web searches; (2) quality of suggested words (3) interaction fluidity of paper browser. According to our on-site observations of the meeting, we found search activities in the meeting were mostly obtrusive, because users had greater tendency to use the given keyboard to make personalized explicit search. Still, each group had at times used words suggested on the semi-ambient surface. According to the subjective feedback in the questionnaire, the words on the table did neither draw their attention nor inspire opportunistic search. Speech only act as secondary input modality, where users sometimes explicitly spoke out the words to be searched for . 7 out of 12 users mentioned that conversational words were useful for forming an intuitive single word search query, but were not useful for forming complex queries; the rest reported that multimedia query suggestions or direct search results may draw more attention from users. This implies the need to reduce the cognitive load of users in the query building process. Regarding the interaction techniques, 10 
out of 12 users reported good experience with paper browsers. The rest complained about the lack of space when all paper browsers are being used at the same time, although this case rarely happened during the experiments. For most of the time, at most 2 paper browsers were active on the table.

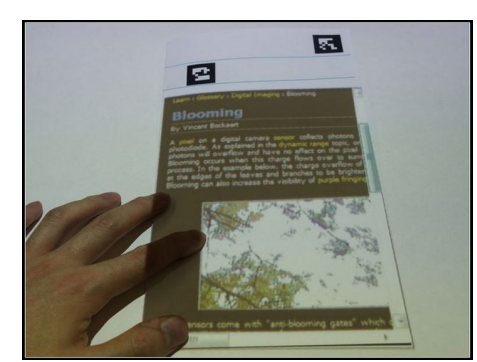

(a) Resizing

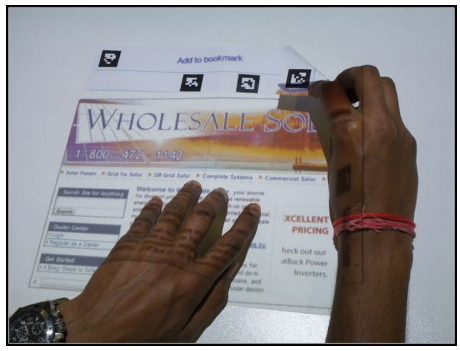

(b) Bookmarking

Figure 4: Folding interactions

\section{Conclusion and Future Work}

We proposed a system setup that combines a semi-ambient informational surface and multiple paper displays to augment collocated meetings with opportunistic search support. The foldable paper browser has achieved satisfaction among users in our study, so that the interaction design have the potential be applied in other similar systems which requires workspace separation and inter-display interaction (paper display interacts with the semi-ambient surface). The result of the informal study showed that spoken words may not have the potential of becoming query suggestion tokens. However, this does not indicate the failure of the "automatic suggestion" concept. The ambient surface is in the users' peripheral view, and they did look at it from time to time during the conversation. We will therefore revise the design of these ambient visualizations by employing more visually-rich content in the semi-ambient surface to tackle the problem, and conversational semantics will be used to provide more intelligent context-aware search recommendations. We also plan to test the system with a real speech recognizer in order to understand the constrains and potential of the technology.

\section{References}

[1] S. Amershi and M. R. Morris. Cosearch: a system for co-located collaborative web search. In Proceeding of
CHI'08, pages 1647-1656.

[2] K. Bachour, F. Kaplan, and P. Dillenbourg. An interactive table for supporting participation balance in face-to-face collaborative learning. Learning Technologies, IEEE Transactions on, 3:203 -213, 2010.

[3] M. R. Morris, J. Lombardo, and D. Wigdor. Wesearch: supporting collaborative search and sensemaking on a tabletop display. In Proceedings of CSCW'10, pages 401-410.

[4] M. R. Morris, A. Paepcke, and T. Winograd. Teamsearch: Comparing techniques for co-present collaborative search of digital media. In Proceedings of TABLETOP'06, pages 97-104.

[5] M. Spindler, S. Stellmach, and R. Dachselt. Paperlens: advanced magic lens interaction above the tabletop. In Proceedings of ITS'09, pages 69-76.

[6] P. Tuddenham, I. Davies, and P. Robinson. Websurface: an interface for co-located collaborative information gathering. In Proceedings of ITS'09, pages 181-188.

[7] G. Zufferey, P. Jermann, and P. Dillenbourg. A tabletop learning environment for logistics assistants: activating teachers. In Proceedings of $\mathrm{HCl}^{\prime} 08$, pages 37-42.

[8] G. Zufferey, P. Jermann, A. Lucchi, and P. Dillenbourg. Tinkersheets: using paper forms to control and visualize tangible simulations. In Proceedings of TEI'09, pages 377-384. 